\title{
SÍNTESIS DE BISMACROMONÓMERO DE 2-OXAZOLINA Y SU POSTERIOR POLIMERIZACIÓN
}

\author{
Juan Carlos Rueda ${ }^{* a}$, Jesús Tito Gutarra ${ }^{a}$
}

\begin{abstract}
RESUMEN
Se investigó la síntesis del bismacromonómero de 2-metil-2-oxazolina (MeOXA) y 2-(2-metoxicarboniletil)-2-oxazolina (EsterOxa) y su posterior copolimerización con $\mathrm{N}$-isopropilacrilamida $\mathrm{y}$ bisacrilamida para formar hidrogeles termosensitivos. El bismacromonómero de 2-oxazolinas fue caracterizado estructuralmente vía resonancia magnética nuclear y se determinó que tuvo un grado de polimerización de 8 unidades y el contenido de EsterOXA fue del $25 \%$ molar.

Los hidrogeles sintetizados fueron hidrolizados y luego fueron introducidos grupos tioles en su estructura. Posteriormente, mediante los grupos tioles, fueron acomplejados iones áuricos, los cuales fueron reducidos dentro de la estructura del hidrogel obteniéndose finalmente nanopartículas de oro en los hidrogeles.

Los hidrogeles fueron caracterizados por su absorción de agua y su sensibilidad a la temperatura en medio acuoso, que se evidenció por una expansión o contracción del volumen del hidrogel debido a la disminución o aumento de la temperatura, respectivamente. Las nanopartículas de oro (NP-Au) contenidas en los hidrogeles, fueron caracterizadas por su resonancia de plasmón superficial a $525 \mathrm{~nm}$ (UV/Vis) y por las fotografías del microscopio electrónico (CEM). Las NP-Au incrementaron la sensibilidad a la temperatura de los hidrogeles y la transición conformacional del mismo ocurrió a mayores temperaturas. Se determinó que los tamaños de las NP-Au variaron entre 5 y $250 \mathrm{~nm}$.
\end{abstract}

Palabras clave: N-isopropilacrilamida, bismacromonómero, 2-oxazolinas, hidrogeles, sensibilidad térmica, nanopartículas de oro.

\section{SYNTHESIS OF 2-OXAZOLINE BISMACROMONOMER AND THEIR POLYMERIZATION}

\begin{abstract}
In this research was studied the synthesis of bismacromonomer of 2-methyl-2-oxazoline (MeOXA) and 2-(2-metoxycarbonylethyl)-2-oxazoline (EsterOxa) and their polymerization
\end{abstract}

a Pontificia Universidad Católica del Perú, jrueda@pucp.edu.pe 
with $\mathrm{N}$-isopropylacrylamide and bisacrylamide for the elaboration of thermosensitive hydrogels. The 2-oxazoline bismacromonomer was structurally characterized by nuclear magnetic resonance (NMR) and was determined that it had a polymerization degree of 8 and the content of EsterOxa was $25 \%$ mol.

The hydrogels were hydrolysed and the generated carboxylic acid groups were transformed into thiol groups which were later complexed with auric ions. The auric ions were reduced and gold nanoparticles (NP-Au) were obtained in situ in hydrogels. The hydrogels were characterized by the water-absorbing capacity and their sensitivity to temperature. This sensitivity was evidenced by the expansion or contraction of hydrogel volume due to the decrease or incresase of temperature, respectively. The gold nanoparticles (NP-Au) content in the hydrogel were characterized by their tipical superficial plasmon resonance at $525 \mathrm{~nm}$ (UV/Vis) and the CEM electron microscopy showed the presence of gold nanoparticles within the hydrogels in a range of sizes from 5 to $250 \mathrm{~nm}$. The NP-Au increased the temperature sensitivity of the hydrogel and the conformational transition occurred at higher temperature.

Key words: N-isopropylacrylamide, bismacromonomer, 2-oxazolines, hydrogels, thermoresponsive hydrogel, gold nanopartícles,

\section{INTRODUCCIÓN}

Las 2-oxazolinas son sustancias heterocíclicas (endoiminoeteres), cuya polimerización ha recibido hasta nuestros días una intensa investigación ${ }^{1-10}$. Las polioxazolinas pueden ser elaboradas a través de una polimerización catiónica por apertura de anillo, la cual es iniciada por grupos electrófilos (ej. ácidos de Brönsted o de Lewis, tosilato y triflato de metilo). Bajo condiciones de reacción apropiadas (alta pureza de monómeros y solventes) la polimerización de las 2-oxazolinas transcurre en una forma "viva", esto quiere decir, sin reacciones de terminación o de transferencia de cadena, y entonces se pueden predeterminar desde el inicio de la polimerización el peso molecular del polímero y su distribución así como su funcionalidad.

Con las 2-oxazolinas se pueden elaborar diferentes arquitecturas poliméricas tales como, por ejemplo, copolímeros en bloque e injertados, macromonómeros e hidrogeles ${ }^{5,8-10}$. La polimetil-, polietil- y poliéster-oxazolina, entre otras, tienen un carácter hidrofílico y con una apropiada selección de los comonómeros se pueden elaborar polímeros amfifílicos.

Las nanopartículas de oro (NP-Au) debido a sus propiedades de inercia, biocompatibilidad, estabilidad y el fenómeno físico de resonancia de plasmón superficial, tienen potencial aplicación en muchos campos tecnológicos, tales como, por ejemplo, electrónica, óptica, biomateriales, catálisis ${ }^{11-13}$. Entre los métodos de síntesis de las NP-Au están el acomplejamiento de iones áuricos con tioles alquílicos y su posterior reducción ${ }^{12}$. 
Nanocompósitos hechos a partir de nanopartículas de oro immersas dentro de hidrogeles tienen posibles aplicaciones en óptica y electrónica. Los hidrogeles pueden posibilitar la formación de NP-Au más estables, con tamaños y morfologías controlados, con tiempos de vida más largos y mayor actividad ${ }^{12,13}$. Langer y colaboradores del Instituto Tecnológico de Massachusetts (USA) elaboraron NP-Au dentro de un hidrogel de poliN-isopropilacrilamida, el cual contenía grupos tioles y disulfuros, los que acomplejaron los iones áuricos y luego estos fueron reducidos para formar finalmente las $\mathrm{NP}-\mathrm{Au}^{12}$. Como resultado se obtuvo una mayor estabilidad de la NP-Au, y se pudo regular el tamaño de las mismas, mediante la variación de la composición del hidrogel. También, debido a que la poliN-isopropilacrilamida tiene la propiedad de tener una transición térmica (low critical solution temperature-LCST) a una determinada temperatura ${ }^{14}$, el hidrogel se contraía y expandía haciendo que las NP-Au se acerquen o alejen entre ellas variando sus propiedades físicas.

En esta investigación hemos sintetizado hidrogeles a partir de bismacromonómeros de 2-oxazolinas e $\mathrm{N}$-isopropilacrilamida. Los hidrogeles obtenidos contenían grupos ácido carboxílicos, los cuales fueron modificados en grupos tioles para acomplejar los iones áuricos y luego de su reducción formar in situ las NP-Au.

\section{PARTE EXPERIMENTAL}

Las sustancias utilizadas en esta investigación fueron obtenidas de las firmas Aldrich y Merck y fueron purificadas según métodos estandar de la literatura ${ }^{1,7-10}$. La 2-metil- y 2-(2-metoxicarboniletil)-2-oxazolina fueron sintetizadas en el laboratorio mediante los procedimientos descritos en la literatura ${ }^{9,10}$.

\section{Síntesis de la 4-vinilbencil-piperazina ("terminador”)}

Procedimiento: en un reactor de vidrio, bajo atmósfera de nitrógeno, se disolvieron 39,6 gramos $(0,46$ moles $)$ de piperazina anhidra en $250 \mathrm{ml}$ de cloroformo seco. Luego, se adicionó a esta solución, a $0^{\circ} \mathrm{C}, 4,6$ gramos $(0,030 \mathrm{~mol})$ de clorometilestireno y trazas de hidroquinona como estabilizador. Después de 10 horas de agitación de esta mezcla a temperatura ambiente, se separaron, mediante filtración, los cristales formados de cloruro de piperazínio. La fase orgánica fue lavada con 5 porciones de $200 \mathrm{ml}$ de agua destilada hasta neutralidad y luego fue secada con sulfato de sodio anhidro, filtrada y reducida en volumen. Finalmente, el producto fue separado del solvente residual mediante evaporación de este y fue obtenido un aceite amarillo-rojizo. Rendimiento 5,7 gramos $(94 \%)^{4}$.

FTIR (KBr): 3390, $3274(\mathrm{NH})$, 3085-3000 (CH, alifático), 2940,2810 (CH, alifático), 1628, $1510,1460(\mathrm{C}=\mathrm{C})$

${ }^{1} \mathrm{H}-\mathrm{RMN}\left(\mathrm{en} \mathrm{CDCl}_{3}\right) \delta: 1.51(\mathrm{NH}) ; 2.40\left(\mathrm{NCH}_{2}\right) ; 2.83\left(\mathrm{NHCH}_{2}\right) ; 3.46\left(\operatorname{ar}-\mathrm{CH}_{2}\right) ; 5.20,5.70$, $6.70\left(\mathrm{ABX}, \mathrm{CH}_{2}=\mathrm{CH}\right) ; 7.30$ (protónes aromáticos).

\section{Síntesis del Bismacromonómero de 2-oxazolinas}

Procedimiento: En un reactor de vidrio seco, bajo atmósfera de nitrógeno seco, se disolvieron 
1,96 g (13,1 mmol) de ioduro de sodio (NaI) en $25 \mathrm{~mL}$ de acetonitrilo seco. Luego, manteniendo el sistema bajo atmósfera de nitrógeno, se adicionaron 3,345 g (39,3 mmol) de 2-metil-2-oxazolina (MeOXA), 2,06 g (13,1 mmol) de 2-(2-metoxicarboniletil)-2-oxazolina (Esteroxa) y finalmente $1,0 \mathrm{~g}(6,55 \mathrm{mmol})$ de clorometilestireno (CMS). El sistema fue calentado a $77^{\circ} \mathrm{C}$ y agitado por siete horas. Finalizado este tiempo, el sistema fue enfriado a temperatura ambiente y se le adicionó una solución del terminador (4-vinilbencilpiperazina) en acetonitrilo y se agitó por una hora. Luego el sistema fue dejado en reposo a $5^{\circ} \mathrm{C}$ por un día. Se eliminaron las sales por filtración, a la solución se le adicionó 4 gramos de carbonato de potasio y se agitó por 12 horas, se filtró y se precipitó el producto polimérico dos veces en éter etílico. Rendimiento 98.5\%. ${ }^{1} \mathrm{H}-\mathrm{RMN}$ : $\delta$ : 1.9-2.2 $\left(\mathrm{CH}_{3}\right) ; 2.5-2.8\left(\mathrm{CH}_{2} \mathrm{CH}_{2} \mathrm{COO}\right)$; 3.4$3.8\left(\mathrm{NCH}_{2} \mathrm{CH}_{2}\right) ; 3.67\left(\mathrm{COOCH}_{3}\right) ; 4.5-4.7\left(\mathrm{Ar}^{-} \mathrm{CH}_{2}\right) ;$ 5.6-6.0 $\left(\mathrm{CH}_{2}=\right) ; 6.7-6.8(\mathrm{CH}=) ; 7.1-7.6$ $\left(\mathrm{H}_{\mathrm{AR}}\right)$.

\section{Síntesis del hidrogel a partir del bismacromonómero de 2-oxazolina}

Procedimiento típico (HG-10): en $3 \mathrm{ml}$ de agua se disolvieron, a $10^{\circ} \mathrm{C}, 0,50 \mathrm{~g}\left(4,4 \times 10^{-4} \mathrm{~mol}\right) \mathrm{de}$ bismacromonómero(TELQ-1)(nohidrolizado), $0,5 \mathrm{~g}\left(4,42 \times 10^{-3} \mathrm{~mol}\right) \mathrm{de} \mathrm{N}$-isopropilacrilamida, $0,02 \mathrm{~g}\left(1,72 \times 10^{-4} \mathrm{~mol}\right)$ de catalizador tetrametiletilendiamina (TEMED). Luego, a esta mezcla fue adicionado $0,020 \mathrm{~g}\left(8,76 \times 10^{-5} \mathrm{~mol}\right)$ de peroxodisulfato de amonio disuelto en $1,0 \mathrm{ml}$ de agua. La mezcla fue agitada y luego fue mantenida en reposo a $10^{\circ} \mathrm{C}$ hasta que se obtuvo el hidrogel, en aproximadamente 15 minutos.

Posteriormente el hidrogel fue purificado mediante lavados, primero con etanol y luego con agua destilada. El hidrogel fue secado hasta peso constante a $35^{\circ} \mathrm{C}$. El rendimiento del hidrogel fue del $98 \%$.

Posteriormente, los grupos ésteres contenidos en el hidrogel fueron hidrolizados in situ mediante una solución de $\mathrm{NaOH} 0,1 \mathrm{~N}$, con un exceso del $100 \%$ molar, respecto del valor estequiométrico. El hidrogel fue embebido en esta solución y calentado a $32^{\circ} \mathrm{C}$ durante 72 horas. Luego, el hidrogel fue lavado con agua destilada hasta $\mathrm{pH}$ neutro. El hidrogel se secó y se guardó en un desecador.

\section{Modificación de los hidrogeles con grupos tioles}

Procedimiento: 0,5 gramos del hidrogel H-HG10 hidrolizado fueron embebidos en $10 \mathrm{ml}$ de agua destilada a $10^{\circ} \mathrm{C}$. A esta mezcla se le agregó 0,10 gramos de EDAC, y 1,10 gramos de sal sódica de N-hidroxisulfosuccinimida (NHS-Na). Se dejó en reposo esta mezcla durante 10 horas. Luego se agregó a la mezcla anterior, 0,065 gramos de hidrocloruro de cisteamina (en exceso estequiométrico). Se dejó reaccionar esta mezcla total por cuatro días en oscuridad y a $5^{\circ} \mathrm{C}$. Luego el hidrogel fue lavado con agua destilada hasta $\mathrm{pH}$ neutro. El hidrogel se secó y se guardó en un desecador hasta su utilización.

\section{Elaboración de nanopartículas de oro dentro del hidrogel modificado}

Se elaboró una solución de una sal de oro, mediante la disolución de 0,020 gramos de la sal HAuCl4 en $200 \mathrm{ml}$ de agua destilada. 0,5 g de hidrogel (ejemplo H-HG10) se colocó en 25 $\mathrm{ml}$ de esta solución por dos días, al cabo de los cuales el hidrogel estaba hinchado o embebido 
de esta solución y había absorbido los iones áuricos tomando una fuerte coloración amarilla. Luego, se preparó una solución de 0,30 gramos de borohidruro de sodio en $100 \mathrm{ml}$ de agua. Con una porción de esta última solución fue realizada la reducción de los iones áuricos dentro del hidrogel. Como producto de este procedimiento se obtuvo nanopartículas de oro dentro de los hidrogeles y se pudo notar que el color del hidrogel cambió de amarillo a violeta oscuro. Las caracterizaciones de los hidrogeles, conteniendo las nanopartículas de oro, se realizó en forma similar a la caracterización de los hidrogeles sin NP-Au.

Prueba de absorción de agua: Se colocó en un vaso una porción de hidrogel hidrolizado seco (peso inicial=Ws) en $80 \mathrm{~mL}$ de agua de destilada a temperatura ambiente durante 24 horas. Pasado este tiempo, se obtuvo el peso del hidrogel hinchado en agua (Wh). El grado de absorción de agua del hidrogel fue calculado mediante la ecuación: $\mathrm{Q}=(\mathrm{Wh}-\mathrm{Ws}) / \mathrm{Ws}$, donde Q es el grado de absorción de agua ( $\mathrm{g} \mathrm{H}_{2} \mathrm{O} / \mathrm{g}$ de $\mathrm{HG}$ seco).

Prueba de sensibilidad a la temperatura: Se determinó la contracción del volumen de los hidrogeles en función de su pérdida de peso y se asumió que el peso es proporcional al volumen. El peso a $25^{\circ} \mathrm{C}$ fue relacionado con el volumen inicial del hidrogel. El porcentaje de contracción, en respuesta al aumento de temperatura, se determinó mediante la ecuación: $\%$ Contracción $=100 *\left(\mathrm{~W}_{\text {inicial }}-\mathrm{W}_{\text {final }}\right) / \mathrm{W}_{\text {inicial }}$

Procedimiento: En un vaso se colocó una porción del hidrogel en $80 \mathrm{~mL}$ de agua a temperatura ambiente por 24 horas. Pasado este tiempo, se sacó el hidrogel del agua y se determinó su peso $\left(\mathrm{W}_{\text {inicial }}\right)$. Seguidamente, la misma porción de hidrogel se sumergió nuevamente en agua y fue colocado en la estufa a una temperatura determinada durante 40 minutos; luego, el hidrogel nuevamente se sacó del agua y se pesó $\left(\mathrm{W}_{\text {final }}\right)$. Se determinó el peso remanente del hidrogel a esta temperatura mediante la ecuación: Peso remanente $(\%)=100 *\left(\mathrm{Peso}_{\text {final }} /\right.$ $\left.\mathrm{Peso}_{\text {inicial }}\right)$. La contracción es la diferencia entre el peso inicial y el peso remanente. Para cada temperatura, se obtuvo un peso remanente $(\%)$ y estos valores fueron ploteados versus la temperatura $\left({ }^{\circ} \mathrm{C}\right)$.

Análisis UV/Visible de los hidrogeles: Se colocó una muestra de hidrogel $(0,3 \mathrm{~g})$ en una celda dentro del espectrómetro Ultravioleta/Visible. Se usó un baño termostatizado con agua destilada para calentar la muestra mediante una chaqueta de calentamiento; las mediciones se iniciaron a $25^{\circ} \mathrm{C}$, con incrementos de $4^{\circ} \mathrm{C}$ cada 20 minutos.

Análisis por microscopía electrónica de barrido (CEM): Las muestras fueron analizadas por la Ing. Kristina Schelestow, en la Universidad Tecnológica de Ilmenau en Alemania, aplicando el procedimiento estándar. 


\section{DISCUSIÓN DE RESULTADOS}

Seguidamente se presentan los resultados de la presente investigación y su análisis respectivo.

Síntesis de la 4-vinilbencilpiperazina ("terminador")

La 4-vinilbencilpiperazina (VBP) se obtuvo a través de la reacción de condensación del clorometilestireno y la piperazina (esquema 1). La piperazina fue usada en la reacción con un exceso molar de 15 a 1 respecto del clorometilestireno para evitar que ocurra la condensación en los dos grupos amina de este compuesto ya que fue necesario preservar uno de ellos para que sirva como agente terminador de la polimerización catiónica de las oxazolinas (síntesis del bismacromonómero).

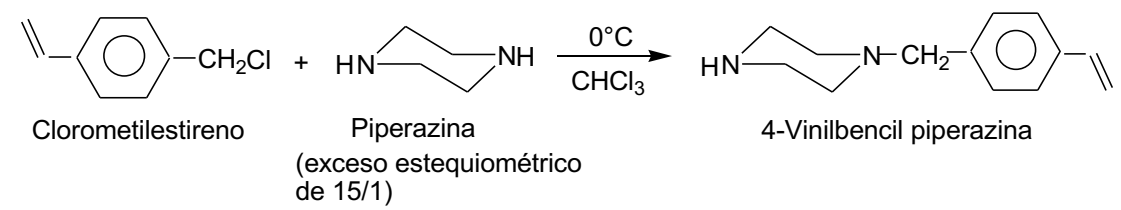

Esquema 1. Síntesis de la 4-vinilbencilpiperazina ("terminador").

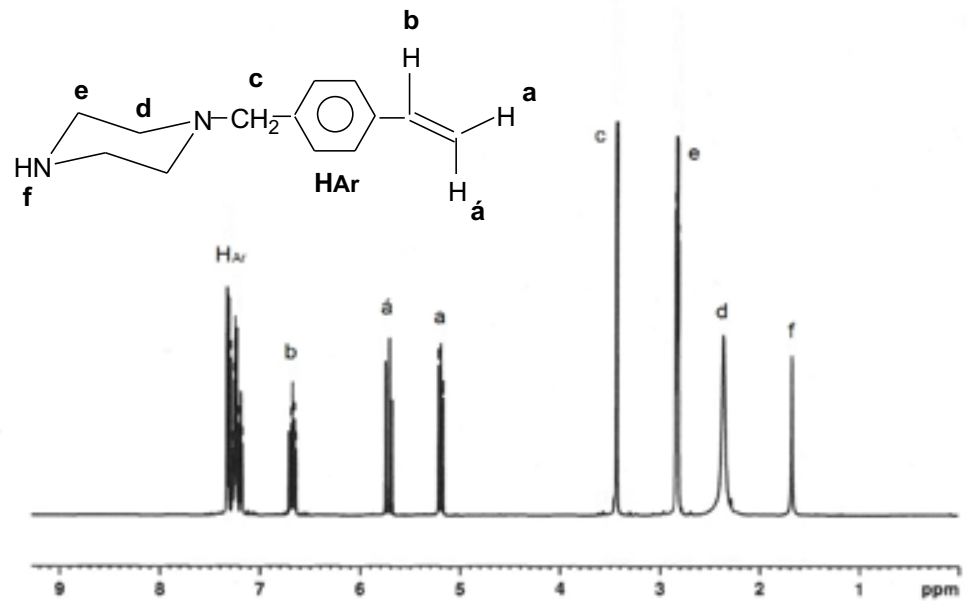

Figura 1. Espectro ${ }^{1} \mathrm{H}-\mathrm{RMN}$ de la 4-vinilbencilpiperazina en cloroformo deuterado a $25^{\circ} \mathrm{C}$.

La 4-vinilbencilpiperazina (VBP) se obtuvo con un rendimiento del 94\%. La VBP fue caracterizada por su espectro de resonancia magnética nuclear e de infrarrojo. A través del anáisis cualitativo y cuantitativo del ${ }^{1} \mathrm{H}-\mathrm{RMN}$ se pudo confirmar la estructura del compuesto sintetizado (Fig. 1). 
Sintesis del bismacromonómero de las 2-oxazolinas

La síntesis del bismacromonómero fue realizada mediante la aplicación simultánea de los métodos del "iniciador" y del "terminador", los cuales son métodos usuales en la síntesis de macromonómeros ${ }^{4}$. La síntesis se realizó mediante la copolimerización catiónica por apertura de anillo de la 2-metil- y de la 2-(2-metoxicarboniletil)-2-oxazolina y fue iniciada por el clorometilestireno (CMS) en presencia del ioduro de sodio (coiniciador). De esta manera, mediante el CMS, se introdujo el grupo vinilo al inicio de la cadena polimérica. La polimerización fue terminada mediante la 4-vinilbencilpiperazina y de esta forma se introdujo el último grupo vinilo en el final de cadena del polímero, obteniéndose así el bismacromonómero (TELQ-1) de las 2-oxazolinas (Esquema 2). La polimerización transcurrió mediante el conocido mecanismo iónico de la polimerización de las 2-oxazolinas 9 .

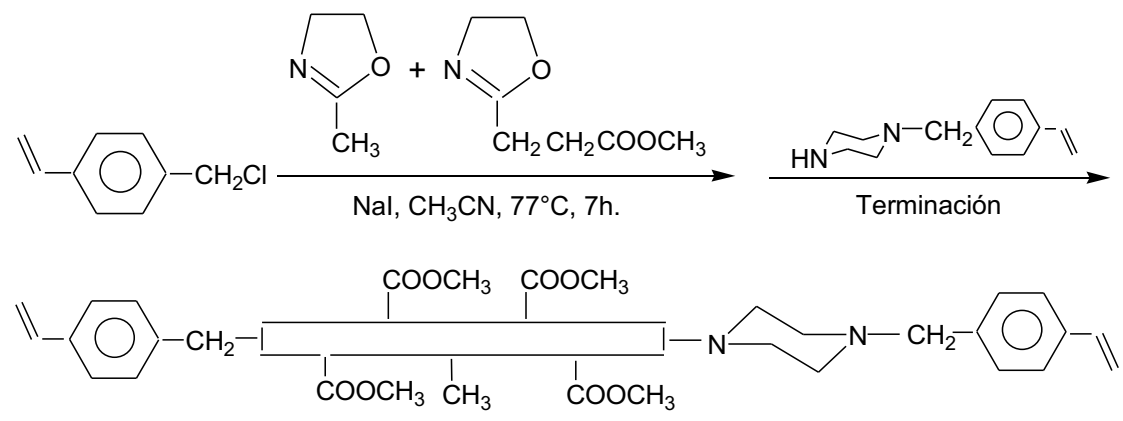

TELQ-1

Esquema 2. Síntesis del bismacromonómero a partir de la polimerización de las 2-oxazolinas.

En la tabla 1 se muestran las condiciones experimentales de síntesis del bismacromonómero y los resultados de la misma.

Tabla 1. Síntesis y caracterización del bismacromonómero TELQ-1

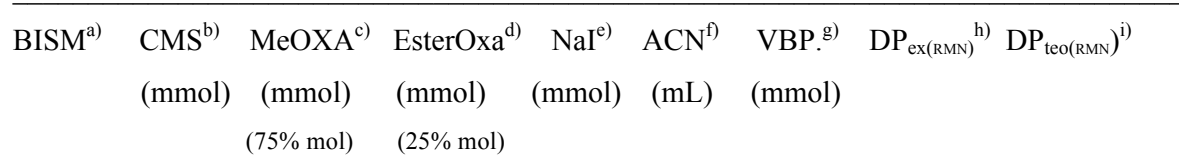

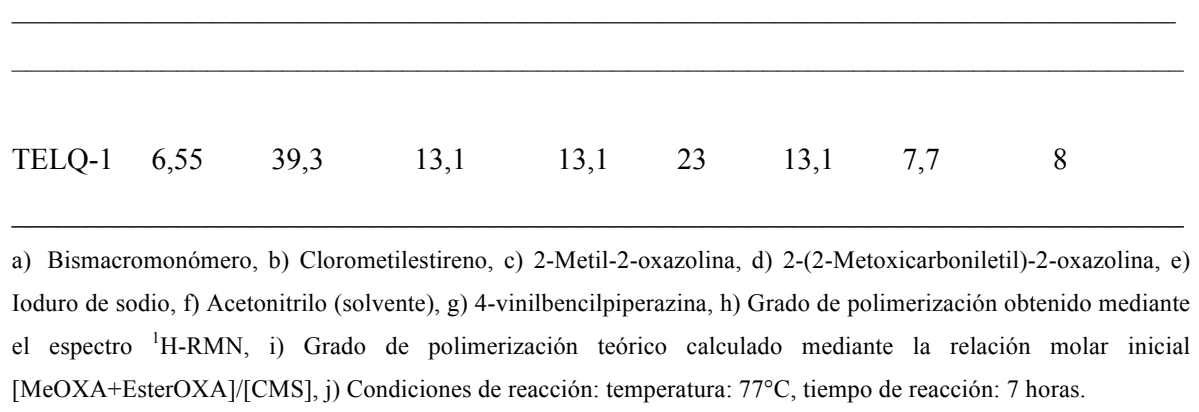


El bismacromonómero TELQ-1 fue obtenido con un rendimiento de1 98,5\% y fue caracterizado mediante resonancia magnética nuclear (figura 2).

Mediante el análisis cuantitativo del espectro ${ }^{1} \mathrm{H}-\mathrm{RMN}$ se determinó que el grado de polimerización (PD) del bismacromonómero fue de 7,7 unidades y la concentración del mero de esteroxazolina en el mismo fue de $25 \%$ molar, lo cual está en excelente concordancia con los valores planeados ( $\mathrm{PD}=8$ y $25 \%$ molar Esteroxa) para esta síntesis (tabla 1). El macromonómero fue soluble en agua y metanol.

Síntesis de los hidrogeles

Fueron sintetizados dos hidrogeles, HG-10 y HG-11 mediante la copolimerización, vía radicales libres, del bismacromonómero TELQ-1 y la N-isopropilacrilamida (NiPAAm) iniciándose la polimerización con el persulfato de amonio y el catalizador Temed a $10^{\circ} \mathrm{C}$ (esquema 3). La gelación fue rápida y ocurrió en solo 15 minutos.

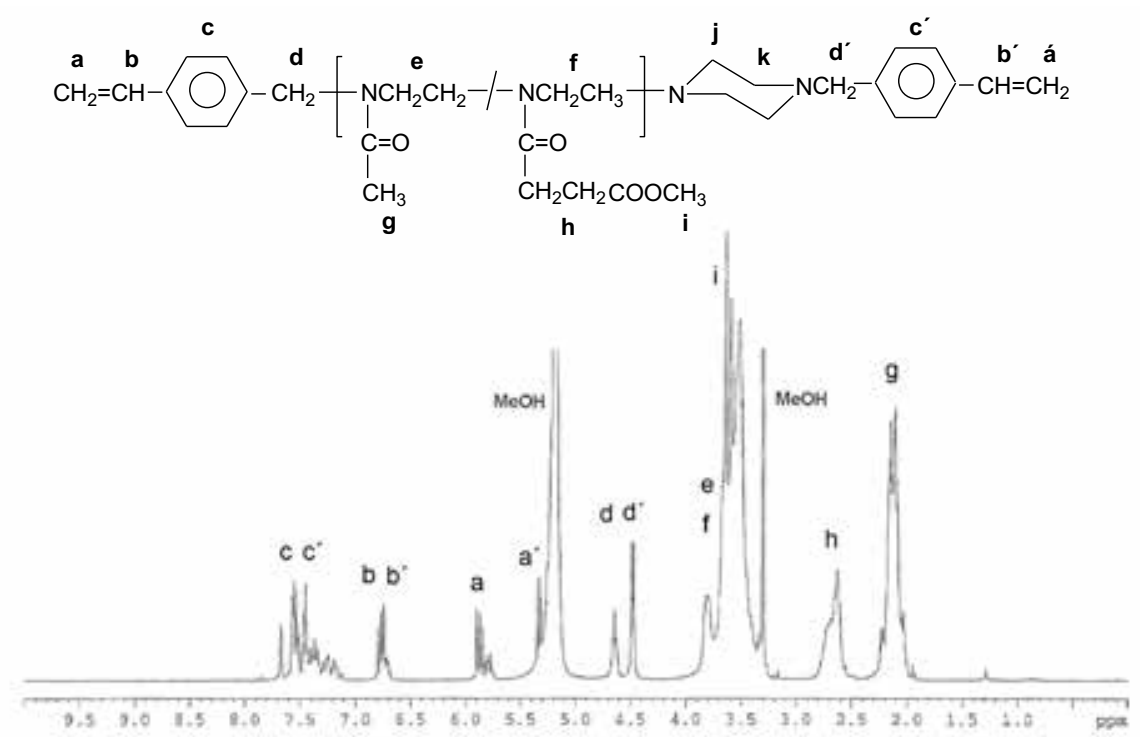

Figura 2. Espectro ${ }^{1} \mathrm{H}-\mathrm{RMN}$ de bismacromonómero TELQ-1 en metanol deuterado a $25^{\circ} \mathrm{C}$ 

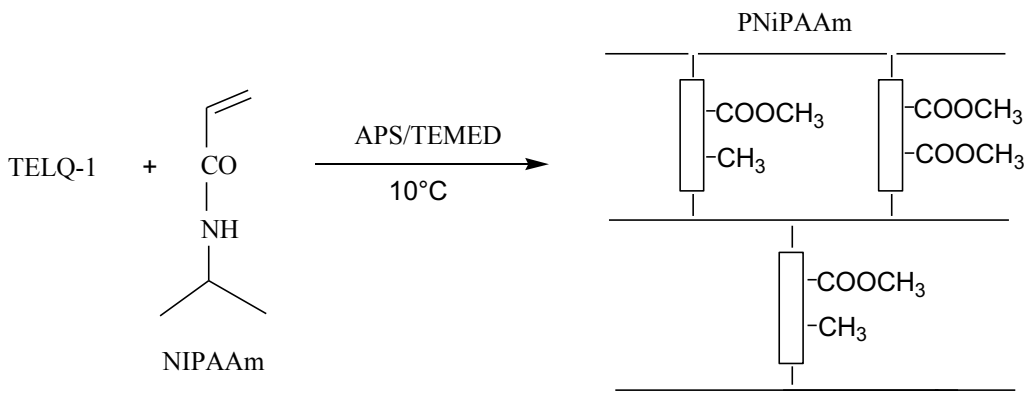

HIDROGEL

Esquema 3. Síntesis del hidrogel a partir de N-isopropilacrilamida y bismacromonómero de 2-oxazolina.

En la síntesis del hidrogel, el bismacromonómero TELQ-1, debido a sus dos grupos vinilicos, actuó como un agente entrecruzador en el sistema de polimerización y por esta razón ya no fue necesario usar un entrecruzador convencional como, por ejemplo, la bisacrilamida.

En la tabla 2 se muestran las condiciones de la síntesis y los resultados obtenidos.

Tabla 2. Elaboración del hidrogel a partir del bismacromonómero y N-isopropilacrilamida.

\begin{tabular}{|c|c|c|c|c|c|c|c|}
\hline Hidrogel & $\begin{array}{c}\left.\text { NiPAAm }^{a}\right) \\
(\mathrm{g})\end{array}$ & $\begin{array}{c}\text { Telq-1 }{ }^{\text {b) }} \\
(\mathrm{g})\end{array}$ & $\begin{array}{c}\operatorname{APS}^{\mathrm{c})} \\
(\mathrm{g})\end{array}$ & $\begin{array}{c}\text { TEMED }^{\mathrm{d})} \\
(\mathrm{g})\end{array}$ & $\begin{array}{c}\text { Solvente }^{\mathrm{e})} \\
(\mathrm{mL})\end{array}$ & $\begin{array}{c}\text { Rend. }{ }^{f)} \\
(\%)\end{array}$ & Abs. Agua ${ }^{g)}$ \\
\hline HG-10 & 0,5 & 0,5 & 0,020 & 0.020 & 4 & 98 & 7,3 \\
\hline HG-11 & 0,5 & 0,30 & 0,020 & 0,020 & 4 & 96 & 7,4 \\
\hline
\end{tabular}

a) N-isopropilacrilamida, b) Bismacromonómero, c) Persulfato de amonio, d) Tetrametiletilendiamina, e) El solvente fue agua destilada f) Rendimiento de la reacción, g) Absorción de agua del hidrogel, en gramos de agua/gramos de hidrogel seco, h) Temperatura de reacción: $10^{\circ} \mathrm{C}$, i) Tiempo de reacción: 24 horas, aunque en 15 minutos ocurrió ya la gelación, se dejó el sistema de reacción por un tiempo de 24 horas antes de iniciar la purificación del hidrogel, 
El rendimiento de los hidrogeles fue relativamente alto, de 98,5 y 97\% para HG-10 e HG-11, respectivamente. Indicando esto que todos los monómeros reaccionaron cuantitativamente. Los grupos éster contenidos en los hidrogeles fueron hidrolizados selectivamente. Esto fue realizado embebiendo a los hidrogeles en una solución de hidróxido de sodio $0,1 \mathrm{~N}$ a $32^{\circ} \mathrm{C}$, durante tres días.

La absorción de agua de los hidrogeles conteniendo grupos éster fue de alrededor del 7,3\%. Sin embargo, luego de la hidrolisis completa de los grupos éster la absorción de agua aumentó hasta 13,1 y 11,9 para los hidrogeles hidrolizados, denominados H-HG-10 y H-HG11, respectivamente. Este aumento del grado de absorción de agua es probablemente debido a que luego de la hidrólisis se generan dentro del hidrogel grupos ácido carboxílicos, más polares que los grupos éster, aumentando así la hidrofilicidad del hidrogel y por consiguiente la absorción de agua.

Los hidrogeles H-HG-10 y H-HG-11, debido a su contenido de segmentos de poliNipAAm, mostraron sensibilidad a la temperatura. Se realizó la prueba de sensibilidad térmica de estos hidrogeles variando la temperatura del agua donde estaban inmersos y se demostró que los hidrogeles se contrajeron al aumentar la temperatura (Fig. 3). Los hidrogeles H-HG-10 y H-HG-11 presentaron valores de transición conformacional a los $47 \mathrm{y} 42^{\circ} \mathrm{C}$, respectivamente y se contrajeron en un 55 y 70\%, respectivamente (Fig. 3). El hidrogel H-HG-10, contiene más grupos funcionales ácido carboxílicos que el H-HG11, y por esto mayor hidrofilicidad, tiene una temperatura de transición mayor y presenta, también por este motivo, una mayor disminución de la intensidad de la transición térmica, tal como ha sido encontrado en sistemas similares ${ }^{5,9,15}$.

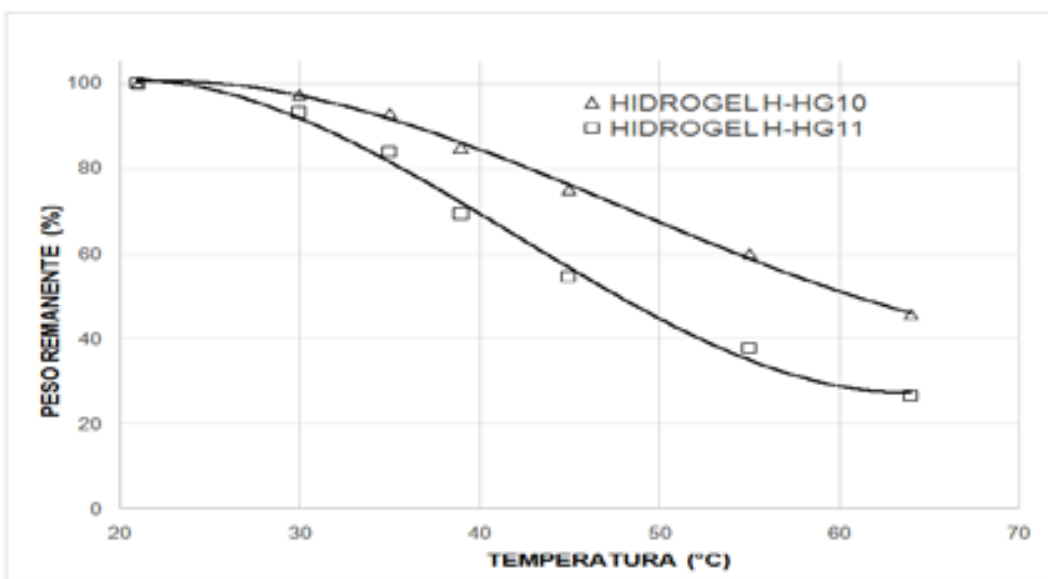

Figura 3. Gráfico de peso remanente vs temperatura para hidrogeles H-HG-10 y H-HG-11 
Modificación de los hidrogeles y obtención de nanopartículas de oro en los mismos

Los hidrogeles H-HG-10 y H-HG-11 fueron modificados en su estructura química. Esto fue realizado mediante la reacción de los grupos ácido carboxílicos del hidrogel con un exceso estequiométrico del hidrocloruro de cisteamina (esquema 4). Se promovió la reacción total del ácido y la amina a baja temperatura mediante el uso de EDAC y la sal sódica de la hidroxisuccinimida. Esta reacción se llevó a cabo en oscuridad, en un rango de $\mathrm{pH}$ entre 4 y 5,5 y a baja temperatura para evitar las reacciones secundarias de degradación de los grupos tioles.

Mediante esta reacción se logró incorporar los grupos tioles en el hidrogel y se asumió, por las condiciones de reacción usadas, que todos los grupos ácidos reaccionaron. Estos grupos tioles sirvieron para acomplejar los iones áuricos cuando el hidrogel se embebió en una solución de $\mathrm{HAuCl}_{4}$. Luego, los iones áuricos fueron entonces reducidos con una solución de borohidruro de sodio para obtener finalmente las nanopartículas de oro dentro de los hidrogeles (esquemas 5 y 6 ).

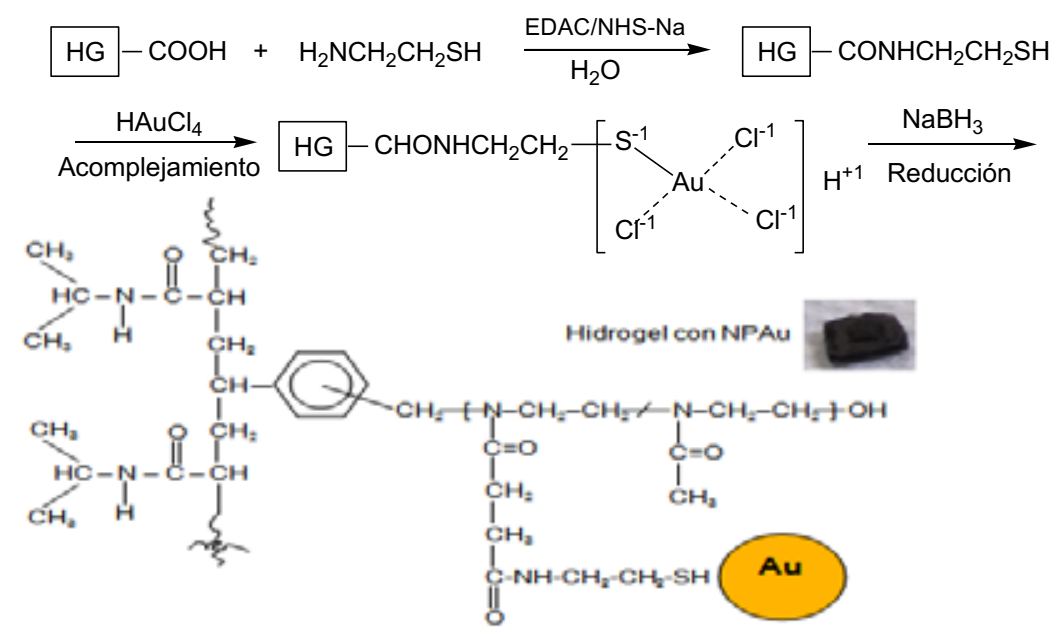

Esquema 4. Modificación de los hidrogeles y obtención de nanopartículas de oro.

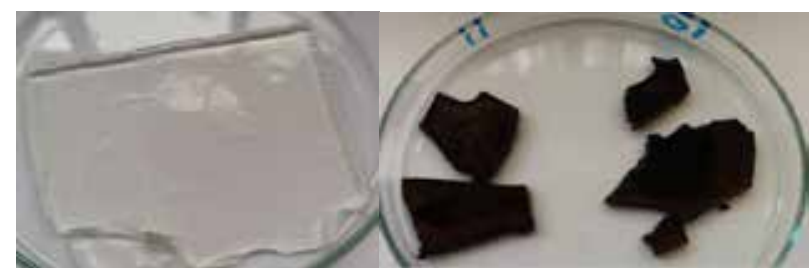

Figura 4. Fotos del hidrogel H-HG10 original sin NP-Au (izquierda) y con NP-Au (derecha). 
Caracterización de los hidrogeles conteniendo nanopartículas de oro

Las NP-Au fueron caracterizadas mediante el análisis ultravioleta/visible. Se obtuvo una curva con un máximo de absorción aproximadamente a $525 \mathrm{~nm}$ que podemos atribuir al típico fenómeno de plasmón superficial de las NP-Au.

Se realizaron pruebas de sensibilidad de los hidrogeles conteniendo iones áuricos y nanopartículas de oro. Los hidrogeles H-HG10 y H-HG11 conteniendo iones áuricos mostraron una mayor sensibilidad a la temperatura que los hidrogeles originales (sin estos iones). Los iones áuricos, en función de su carga eléctrica, podrían formar interacciones con los segmentos poliméricos polares de poliNIPAAm y del bismacromonómero y al ocurrir el colapso del poliNiPAAm (al alcanzarse la temperatura de transición conformacional) los iones áuricos entonces ayudarían a un colapso más eficiente o masivo de toda la masa del hidrogel (Figs. 5 y 6).

El hidrogel H-HG10 conteniendo nanopartículas de oro, al aumentar la temperatura, mostró una mayor contracción de su volumen así como su temperatura de transición conformacional fue mayor que el hidrogel original. Con el hidrogel H-HG11 se obtuvo el resultado contrario. El hidrogel H-HG11, conteniendo NP-Au, mostró una ligera menor contracción que el hidrogel original. Podemos asumir que en los hidrogeles, las nanopartículas de oro estarían localizadas cerca de las unidades de bismacromonómero (reticulador) porque fueron estos los elementos que contenían los grupos tioles y ahí se redujeron los iones áuricos para formar las NP-Au. Las NP-Au podrían ejercer el efecto de ayudar efectivamente en el colapso total del hidrogel si existen en este en una proporción relativamente alta como en el caso H-HG10 y en el caso del hidrogel H-HG11, no se alcanzaría este umbral de concentración de NP-Au para surtir el mismo efecto.

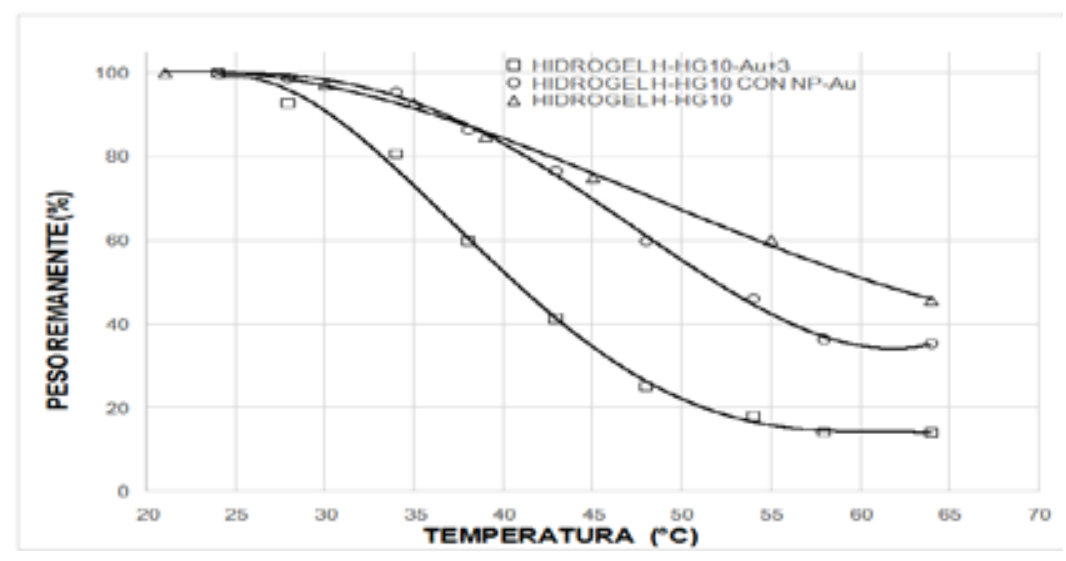

Figura 5. Gráfico de peso remanente vs temperatura para $\mathrm{H}-\mathrm{HG}-\mathrm{G} 10, \mathrm{H}-\mathrm{HG} 10+\mathrm{Au}^{+3}$, e H-HG-10-NP-Au $\left(\mathrm{Au}^{+3}\right.$ reducido con $\left.\mathrm{BH}_{3}\right)$. 
Por otro lado, se determinó, mediante medición por el microscopio electrónico de barrido CEM de la Universidad Tecnológica de Ilmenau, que efectivamente se formaron nanopartículas de oro y que su rango de tamaños fue de 5 a $250 \mathrm{~nm}$ (figura 7).

El objetivo principal de obtener un hidrogel termosensitivo en base a bismacromonómero de 2-oxazolinas funcionalizadas y que pueda contener nanopartículas de oro se ha cumplido. La variación del volumen del hidrogel podría variar las propiedades físicas de las nanopartículas de oro mediante su acercamiento (contracción del hidrogel) o alejamiento (expansión del hidrogel).

La investigación de la variación de las propiedades físicas será materia de un estudio posterior.

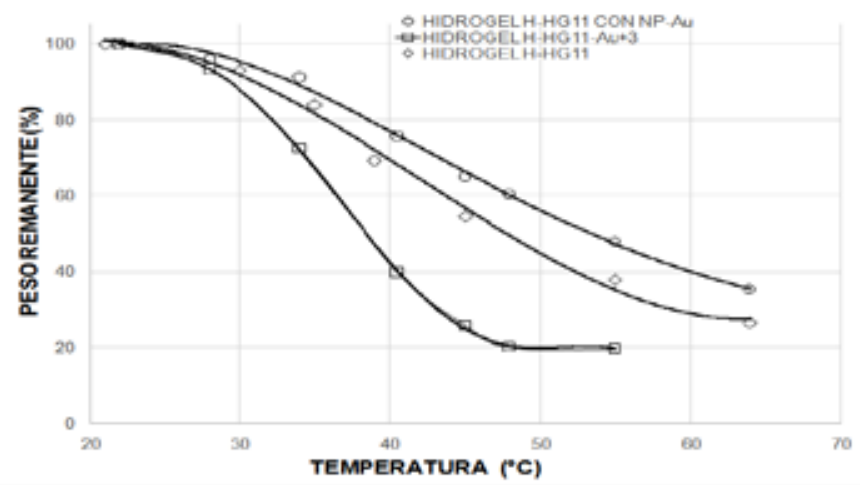

Figura 6. Gráfico de peso remanente vs temperatura para $\mathrm{H}-\mathrm{HG}-\mathrm{G} 11, \mathrm{H}-\mathrm{HG} 11+\mathrm{Au}^{+3}$, e H-HG-11-NP-Au $\left(\mathrm{Au}^{+3}\right.$ reducido con $\left.\mathrm{BH}_{3}\right)$.

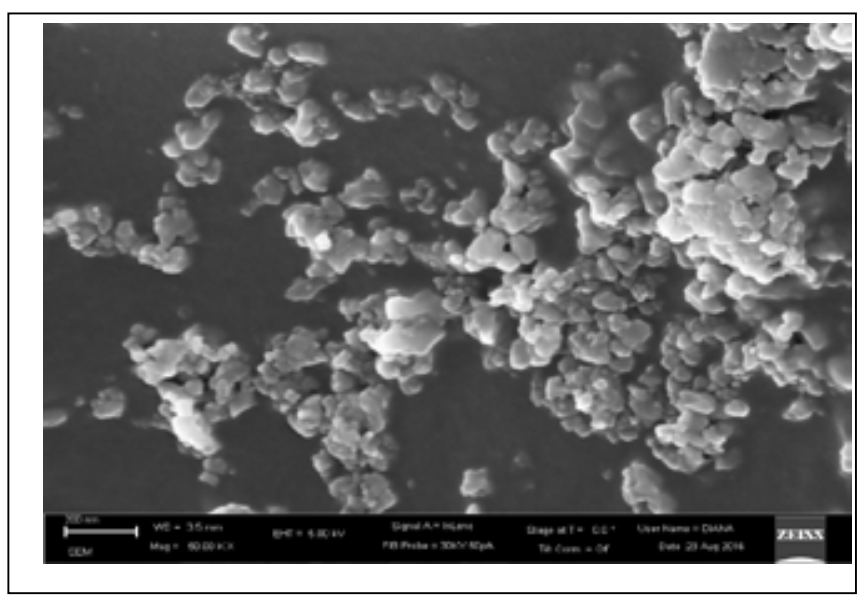

Figura 7. Microfotografias del hidrogel H-HG-10 conteniendo nanopartículas de oro mediante el microscopio electrónico de barrido (Universidad Tecnológica de Ilmenau, Alemania) 


\section{CONCLUSIONES}

Es factible la síntesis del bismacromonómero de 2-oxazolinas mediante la polimerización catiónica por apertura de anillo de estos monómeros, iniciada por el clorometilestireno y terminada por la 4-vinilbencilpiperazina.

Es factible la elaboración de un hidrogel mediante la polimerización de la $\mathrm{N}$-isopropilacrilamida y utilizando el bismacromonómero de 2-oxazolinas como un elemento entrecruzador.

Las nanopartículas de oro generadas dentro de los hidrogeles aumentan la sensibilidad térmica del hidrogel respecto del hidrogel sin NP-Au. Se observó una contracción y expansión del hidrogel conteniendo las nanopartículas de oro, lo que podría hacer variar las propiedades ópticas y eléctricas de estas.

Los iones áuricos, cuando están acomplejados dentro de hidrogeles de poliNIPAAm, provocan el aumento de la sensibilidad térmica del hidrogel y también un aumento de la intensidad del fenómeno de transición conformacional.

\section{AGRADECIMIENTOS}

A la Pontificia Universidad Católica del Perú (PUCP) por el financiamiento de esta investigación, al Dr. Hartmut Komber, del Instituto Leibniz de Investigaciones en Polímeros de Dresden, Alemania, por los análisis de resonancia magnética nuclear y a la Ing. Kristina Schelestow por los análisis de microscopía electrónica CEM en la Universidad Tecnológica de Ilmenau, Alemania.

\section{BIBLIOGRAFÍA}

1. Bloksma M, Weber C, Perevyazko I, Kuse A, Hoogenboom R, Schubert U. Poly(2cyclopropyl-2-oxazoline): From Rate Acceleration by Cyclopropyl to Thermoresponsive Properties. Macromolecules. 2011; 44, 4057-4064.

2. Bloksma M, Schubert U, Hoogenboom R. Poly(cyclic imino ether)s Beyond 2-Substituted-2-oxazolines. Macromol Rapid Commun. 2011; 32: 1419-1441.

3. Hoogenboom R, Fitjen MWM, Schubert US. Parallel kinetic investigation of 2-oxazoline polymerizations with different initiators as basis for designed copolymer synthesis. $\mathrm{J}$ Polym Sci. Part A: Polym Chem. 2004; 42: 1830-1840.

4. Rueda JC, Komber H, Cedrón JC, Voit B, Shevtsova G. Synthesis of New Hydrogels by Copolymerization of Poly(2-methyl-2-oxazoline) Bis(macromonomers) and N-Vinylpyrrolidone. Macromol Chem Phys. 2003, 204: 947-953.

5. Rueda JC, Zschoche S, Komber H, Schmaljohann D, Voit B. Synthesis and Characterization of Thermoresponsive Graft Copolymers of NIPAAm and 2-Alkyl-2oxazolines by the "Grafting from" Method. Macromolecules. 2005; 38: 7330-7336. 
6. Rueda JC, Zschoche S, Komber H, Krahl F, Arndt K-F, Voit B. New Thermo-Sensitive Graft Copolymers Based on a Poly(N-isopropylacrylamide) Backbone and Functional Polyoxazoline Grafts with Random and Diblock Structure. Macromol Chem Phys. 2010; 211: 706-711.

7. Zschoche S, Rueda JC, Binner M, Komber H, Janke A, Arndt K-F, Voit B. Macromol Chem Physic. Reversibly Switchable $\mathrm{pH}$ - and Thermoresponsive Core-Shell Nanogels Based on Poly(NiPAAm)-graft-poly(2-carboxyethyl-2-oxazoline)s. 2012; 213: 215226.

8. Zarka T, Nuyken O, Weberskirch R. Amphiphilic Polymer Supports for the Asymmetric Hydrogenation of Amino Acid Precursors in Water. Chem Eur J. 2003; 9: 3228-3234.

9. Rueda JC, Campos E, Komber H, Zschoche S, Häussler L, Voit B. Synthesis and Characterization of New $\mathrm{pH}$ - and Thermoresponsive Hydrogels Base on N-Isopropylacrylamide and 2-Oxazolines. Des Monomers Polym. . 2014; 17: 208-216.

10. Zschoche S, Rueda JC, Binner M, Komber H, Janke A, Appelhans D, Voit B. Temperature- and $\mathrm{pH}$-dependent aggregation behaviour of hydrophilic dual-sensitive poly(2-oxazoline)s block copolymers as latent amphiphilic macromolecules. European Polymer Journal. 2017; 88: 623-635.

11. Daniel MC, Astruc D. Gold nanoparticles: assembly, supramolecular chemistry, quantum-size-related properties, and applications toward biology, catalysis, and nanotechnology. Chem Rev. 2004; 104: 293-346.

12. Wang C, Flynn N, Langer R. Controlled structure and properties of thermoresponsive nanoparticle-hydrogel composites. Advanced Materials. 2004; 16: 1074-1079.

13. Pong FY, Lee M, Bell JR, Flynn NT. Thermoresponsive Behavior of Poly(NIsopropylacrylamide) Hydrogels Containing Gold Nanostructures. Langmuir. 2006; 22: 3851-3857.

14. Schild HG. Poly(N-isopropylacrylamide): experiment, theory and application. Prog Polym Sci. 1992; 17: 163-249.

15. Dimitrov I, Trzebicka B, Müller A, Dworak A, Tsvetanov C. Thermosensitive watersoluble copolymers with doubly responsive reversibly interacting entities. Prog Polym Sci. 2007; 32: 1275-1343 